\title{
AN APPLICATION OF A NONCOMPACTNESS TECHNIQUE TO AN INVESTIGATION OF THE EXISTENCE OF SOLUTIONS TO A NONLOCAL MULTIVALUED DARBOUX PROBLEM
}

\author{
LUDWIK BYSZEWSKI \\ Cracow University of Technology \\ Institute of Mathematics \\ Warszawska 24 \\ 31-155 Cracow, Poland \\ NIKOLAOS S. PAPAGEORGIOU \\ National Technical University \\ Department of Mathematics \\ Zografou Campus \\ Athens 15773, Greece
}

(Received January, 1998; Revised June, 1998)

The aim of the paper is to prove two theorems on the existence of solutions to a nonlocal multivalued Darboux problem. The first theorem concerns the case when the orientor field is convex valued. The second theorem concerns the case when the orientor field is nonconvex valued. A compactness type condition involving the ball measure of noncompactness is applied.

Key words: Multivalued Darboux Problem, Nonlocal Conditions, Measure of Noncompactness, Measurable Multifunctions, Upper and Lower Semicontinuous Multifunctions, Fixed Point Principle.

AMS subject classifications: 35R70, 35L70, 35L15, 35L99.

\section{Introduction}

In this paper we study the existence of solutions to a nonlocal multivalued Darboux problem in a separable Banach space. Applying a compactness type condition involving the ball measure of noncompactness, we obtain two theorems on the existence of the solutions of the nonlocal multivalued Darboux problem. The first theorem concerns the case when the orientor field is convex valued. The second theorem concerns the case when the orientor field is nonconvex valued. 
The results obtained are generalizations of those given by Papageorgiou in [13] on the existence of solutions of a classical multivalued Darboux problem. They are also generalizations of those given by Byszewski in $[3,4,6]$ and by Byszewski and Lakshmikantham in [5] on the existence and uniqueness of solutions of nonlocal Darboux problems.

The approach applied in the paper is based on results of Papageorgiou [11-13], Kandilakis and Papageorgiou [8, 9], and Byszewski $[3,4,6]$.

The existence of a solution of a classical multivalued problem, where the orientor field has compact values in a separable Banach space, was also examined by Dawidowski and Kubiaczyk in [7] using a contraction principle for multifunctions.

\section{Preliminaries}

Let $(\Omega, \Sigma)$ be a measurable space and $Y$ a separable Banach space. We will need the following sets:

and

$$
P_{f(c)}(Y):=\{A \subset Y: A \text { is nonempty, closed, (convex) }\}
$$

$$
P_{(w) k(c)}(Y):=\{A \subset Y: A \text { is nonempty, (weakly-) compact, (convex) }\} .
$$

A multifunction $F: \Omega \rightarrow P_{f}(Y)$ is said to be measurable if there exists a sequence of measurable functions $f_{n}: \Omega \rightarrow Y(n \in \mathbb{N})$ such that $F(\omega)={\overline{\left\{f_{n}(\omega)\right\}}}_{n \in \mathbb{N}}$ for all $\omega \in \Omega$.

The multifunction $F$ is said to be weakly measurable if for every $y^{*} \in Y^{*}$ the $\mathbb{R}=$ $\mathbb{R} \cup\{\infty\}$-valued function $\omega \rightarrow \sigma\left(y^{*}, F(\omega)\right)=\sup \left\{\left(y^{*}, y\right): y \in F(\omega)\right\}$ is measurable.

Let $\mathscr{B}$ be the family of bounded subsets of $Y$. Then the ball measure of noncompactness $\beta: \mathfrak{B} \rightarrow[0, \infty)$ is defined by

$$
\begin{gathered}
\beta(B):=\inf \{\rho>0: B \text { can be covered by finitely many balls of the radii } \rho\}, \\
B \in \mathscr{B} .
\end{gathered}
$$

Let $Y_{i}(i=1,2)$ be Hausdorff topological spaces.

A multifunction $G: Y_{1} \rightarrow 2^{Y_{2}} \backslash\{\emptyset\}$ is said to be upper semicontinuous [respectively, lower semicontinuous] if for every open set $A \subset Y_{2},\left\{y \in Y_{1}: G(y) \subset A\right\}$ [respectively, $\left.\left\{y \in Y_{1}: G(y) \cap A \neq \emptyset\right\}\right]$ is open in $Y_{1}$.

We will need the sets $\mathbb{R}_{+}:=(0, \infty)$ and $\Delta:=I \times I$, where $I:=[0, c]$ and $c>0$.

Let $X:=C(I, E)$, where $E$ is a separable Banach space with the norm $\|\cdot\|$, and let $C$ be the Banach space defined by

$$
C:=\{(\tilde{\phi}, \tilde{\psi}) \in X \times X: \tilde{\phi}(0)=\tilde{\psi}(0)\}
$$

and equipped with the norm $\|\cdot\|_{C}$ given by the formula

$$
\|(\tilde{\phi}, \tilde{\psi})\|_{C}:=\|\tilde{\phi}\|_{X}+\|\tilde{\psi}\|_{X}, \quad(\tilde{\phi}, \tilde{\psi}) \in C .
$$

By $E_{w}$ we denote the Banach space $E$ with the weak topology.

Let $p$ and $r$ be arbitrary natural numbers. 
For given a multifunction $F: \Delta \times E \rightarrow P_{f c}(E)\left[F: \Delta \times E \rightarrow P_{f}(E)\right.$, respectively $]$ and, given a $(\phi, \psi) \in C$, satisfying some assumptions, we will study the existence of a solution of the following Darboux problem:

$$
\left.\begin{array}{c}
\frac{\partial^{2} u(x, y)}{\partial x \partial y} \in F(x, y, u(x, y)) \text { a.e. in } \Delta, \\
u(x, 0)+\sum_{j=1}^{r} h_{j}(x) u\left(x, b_{j}\right)=\phi(x), \quad x \in I, \\
u(0, y)+\sum_{i=1}^{p} k_{i}(y) u\left(a_{i}, y\right)=\psi(y), \quad y \in I,
\end{array}\right\}
$$

where $a_{i}(i=1, \ldots, p)$ and $b_{j}(j=1, \ldots, r)$ are given numbers such that

and

$$
0<a_{1}<\ldots<a_{p} \leq c
$$

$$
0<b_{1}<\ldots<b_{r} \leq c .
$$

A function $u \in C(\Delta, E)$ is said to be a solution of problem (2.2) if there is $f \in$ $L^{1}(\Delta, E)$ such that

and

$$
f(\xi, \eta) \in F(\xi, \eta, u(\xi, \eta)) \text { a.e. in } \Delta
$$

where

$$
\begin{aligned}
u(x, y)= & \alpha(x, y)-\sum_{j=1}^{r} h_{j}(x) u\left(x, b_{j}\right)-\sum_{i=1}^{p} k_{i}(y) u\left(a_{i}, y\right) \\
& +\int_{0}^{x} \int_{0}^{y} f(\xi, \eta) d \xi d \eta, \quad(x, y) \in \Delta
\end{aligned}
$$

$$
\alpha(x, y):=\phi(x)+\psi(y)-\phi(0), \quad(x, y) \in \Delta .
$$

Let $Z$ be a fixed nonempty compact subset of $E$.

By $K(\Delta, E)$ we denote the set of the functions $w$ belonging to $C(\Delta, E)$ such that

$$
w(x, 0)+w(0, y) \in Z \text { for }(x, y) \in \Delta \text {. }
$$

\section{Theorem About the Existence of a Solution of the Nonlocal Multi- valued Darboux Problem with the Convex Valued Orientor Field}

Theorem 3.1: Assume that $F: \Delta \times E \rightarrow P_{f c}(E)$ is a multifunction such that:

(i) $\quad(x, y, z) \rightarrow F(x, y, z)$ is weakly measurable, $z \rightarrow F(x, y, z)$ is upper semicontinuous from $E$ into $E_{w}$ and for all $(x, y) \in \Delta, F(x, y, \cdot)$ maps the bounded sets into relatively weakly compact sets,

(ii) $|F(x, y, z)|:=\sup \{\|s\|: s \in F(x, y, z)\}$

$$
\leq c_{1}(x, y)+c_{2}(x, y)\|z\| \text { a.e., }
$$


where $c_{i}(i=1,2)$ are some functions belonging to $L^{1}\left(\Delta, \mathbb{R}_{+}\right)$, and

$$
\beta(F(x, y, B)) \leq \mathfrak{S}(x, y) \beta(B) \text { a.e. }
$$

for every nonempty and bounded set $B \subset E$, where $\mathscr{G}$ is a function belonging to $L^{\infty}\left(\Delta, \mathbb{R}_{+}\right)$.

Moreover, assume that

(iii) $\phi, \psi \in C$,

(iv) $\quad h_{j} \in C(I, \mathbb{R}) \quad(j=1, \ldots, r), \quad k_{i} \in C(I, \mathbb{R}) \quad(i=1, \ldots, p), \quad h_{j}(0)=0 \quad(j=$ $1, \ldots, r), k_{i}(0)=0(i=1, \ldots, p)$ and

$$
\left(\sum_{j=1}^{r}\left\|h_{j}\right\|_{C(I, \mathbb{R})}+\sum_{i=1}^{p}\left\|k_{i}\right\|_{C(I, \mathbb{R})}\right) e^{\left\|c_{2}\right\|} L^{1}(\Delta, \mathbb{R}+)<1
$$

Then in a class of functions $w \in K(\Delta, E)$ problem (2.2) possesses a solution.

Proof: Firstly, we will obtain an a priori evaluation for the solutions of problem (2.2). For this purpose suppose that $u$ is a solution of problem (2.2). Consequently, we have, from (2.3)-(2.5), that

$$
\begin{gathered}
\|u(x, y)\| \leq\|\alpha(x, y)\|+\sum_{j=1}^{r}\left|h_{j}(x)\right|\left\|u\left(x, b_{j}\right)\right\|+\sum_{i=1}^{p}\left|k_{i}(y)\right|\left\|u\left(a_{i}, y\right)\right\| \\
+\int_{0}^{x} \int_{0}^{y}\|f(\xi, \eta)\| d \xi d \eta, \quad(x, y) \in \Delta .
\end{gathered}
$$

Formulas (3.4), (2.3) and (3.1) imply that

$$
\begin{aligned}
\|u(x, y)\| \leq \alpha_{0} & +c_{h, k}\|u\|_{C(\Delta, E)}+\int_{0}^{x} \int_{0}^{y}\left[c_{1}(\xi, \eta)+c_{2}(\xi, \eta)\|u(\xi, \eta)\|\right] d \xi d \eta \\
& \leq \alpha_{0}+c_{h, k}\|u\|_{C(\Delta, E)}+\left\|c_{1}\right\|_{L^{1}\left(\Delta, \mathbb{R}_{+}\right)} \\
& +\int_{0}^{x} \int_{0}^{y} c_{2}(\xi, \eta)\|u(\xi, \eta)\| d \xi d \eta, \quad(x, y) \in \Delta
\end{aligned}
$$

where

$$
\alpha_{0}:=\|\phi\|_{X}+\|\psi\|_{X}+\|\phi(0)\|
$$

and

$$
c_{h, k}:=\sum_{j=1}^{r}\left\|h_{j}\right\|_{C(I, \mathbb{R})}+\sum_{i=1}^{p}\left\|k_{i}\right\|_{C(I, \mathbb{R})^{\circ}}
$$

By (3.5) and Gronwall's inequality,

$$
\|u(x, y)\| \leq\left(\alpha_{0}+c_{h, k}\|u\|_{C(\Delta, E)}+\left\|c_{1}\right\|_{L^{1}\left(\Delta, \mathbb{R}_{+}\right)}\right) e^{\left\|c_{2}\right\|_{L^{1}\left(\Delta, \mathbb{R}_{+}\right)}}
$$


From (3.6), we obtain

$$
\begin{gathered}
\left(1-c_{h, k} e^{\left\|c_{2}\right\|} L^{1}\left(\Delta, \mathbb{R}_{+}\right)\right)\|u\|_{C(\Delta, E)} \\
\leq\left(\alpha_{0}+\left\|c_{1}\right\| L_{L^{1}\left(\Delta, \mathbb{R}_{+}\right)}\right) e^{\left\|c_{2}\right\|} L^{1}\left(\Delta, \mathbb{R}_{+}\right) .
\end{gathered}
$$

Consequently, by (3.7) and (3.3),

$$
\|u(x, y)\| \leq \frac{\alpha_{0}+\left\|c_{1}\right\|_{L^{1}\left(\Delta, \mathbb{R}_{+}\right)}}{1-c_{h, k} e^{\left\|c_{2}\right\|_{L^{1}\left(\Delta, \mathbb{R}_{+}\right)}}} e^{\left\|c_{2}\right\|_{L^{1}\left(\Delta, \mathbb{R}_{+}\right)}},(x, y) \in \Delta .
$$

Then, from (3.8),

$$
\|u(x, y)\| \leq M, \quad(x, y) \in \Delta
$$

where

$$
M:=\frac{\alpha_{0}+\left\|c_{1}\right\|}{L^{1}\left(\Delta, \mathbb{R}_{+}\right)} e^{\left\|c_{2}\right\|_{L^{1}\left(\Delta, \mathbb{R}_{+}\right)}{ }^{1-c_{h, k} e^{1}\left(\Delta, \mathbb{R}_{+}\right)}}
$$

Define $\widehat{F}: \Delta \times E \rightarrow P_{f c}(E)$ by the formula

$$
\widehat{F}(x, y, z):=\left\{\begin{array}{cl}
F(x, y, z) & \text { if }\|z\| \leq M \\
F\left(x, y, \frac{M z}{\|z\|}\right) & \text { if }\|z\|>M
\end{array}\right.
$$

where $M$ is given by $(3.10)$.

It follows, from (3.11), that

$$
\widehat{F}(x, y, z)=F\left(z, y, p_{M}(z)\right) \text { in } \Delta \times E,
$$

where $p_{M}$ is the $M$-radial retraction in $E$.

Since $p_{M}$ is Lipschitz continuous then (3.11) and the first part of assumption $(i)$ imply that $(x, y, z) \rightarrow \widehat{F}(x, y, z)$ is weakly measurable. Moreover, from (3.11), the second part of assumption $(i)$ and Theorem 7.3.11 from [10], $z \rightarrow \widehat{F}(x, y, z)$ is upper semicontinuous from $E$ into $E_{w}$. By (2.1), (3.12), (3.2) and by the inclusion,

$$
p_{M}(B) \subset \overline{\operatorname{conv}}(B \cup\{0\}), \quad B \subset E,
$$

we have

$$
\begin{gathered}
\beta(\widehat{F}(x, y, B))=\beta\left(F\left(x, y, p_{M}(B)\right)\right) \leq \mathscr{S}(x, y) \beta\left(p_{M}(B)\right) \\
\leq \mathfrak{S}(x, y) \beta(\overline{\operatorname{conv}}(B \cup\{0\})) \leq \mathscr{S}(x, y) \beta(B) \text { a.e. in } \Delta, B \subset E .
\end{gathered}
$$


Observe that, from (3.11) and (3.1),

$$
\begin{gathered}
|\widehat{F}(x, y, z)|=\sup \{\|s\|: s \in \widehat{F}(x, y, z)\} \\
\leq c_{1}(x, y)+M c_{2}(x, y)=: c_{3}(x, y) \text { a.e. with } c_{3} \in L^{1}\left(\Delta, \mathbb{R}_{+}\right) .
\end{gathered}
$$

Define a set $\mathcal{U}$ by

$$
\begin{aligned}
& \text { น: }=\{u \in C(\Delta, E): u(x, 0)+u(0, y) \in Z,(x, y) \in \Delta, \\
& u(x, y)=\alpha(x, y)-\sum_{j=1}^{r} h_{j}(x) u\left(x, b_{j}\right)-\sum_{i=1}^{p} k_{i}(y) u\left(a_{i}, y\right) \\
& +\int_{0}^{x} \int_{0}^{y} g(\xi, \eta) d \xi d \eta, \quad(x, y) \in \Delta \\
& \left.\|g(\xi, \eta)\| \leq c_{3}(\xi, \eta) \text { a.e. in } \Delta\right\}
\end{aligned}
$$

and a multifunction

$$
T: \varkappa \rightarrow 2 \text { น }
$$

by

$$
\begin{gathered}
T(u):=\left\{v \in C(\Delta, E): v(x, y)=\alpha(x, y)-\sum_{j=1}^{r} h_{j}(x) u\left(x, b_{j}\right)-\sum_{i=1}^{p} k_{i}(y) u\left(a_{i}, y\right)\right. \\
+\int_{0}^{x} \int_{0}^{y} f(\xi, \eta) d \xi d \eta, \quad(x, y) \in \Delta, \\
\left.f \in L^{1}(\Delta, E), f(\xi, \eta) \in \widehat{F}(\xi, \eta, u(\xi, \eta)) \text { a.e. in } \Delta\right\}, u \in \text { U. }
\end{gathered}
$$

Since $(\xi, \eta, z) \rightarrow \widehat{F}(\xi, \eta, z)$ is weakly measurable, $(\xi, \eta) \rightarrow \widehat{F}(\xi, \eta, u(\xi, \eta))$ is weakly measurable on $\Delta$ with the Lebesgue $\sigma$-field which is complete with respect to the Lebesgue measure on $\Delta$. So, $(\xi, \eta) \rightarrow \widehat{F}(\xi, \eta, u(\xi, \eta))$ is measurable and, therefore, by Aumann's selection theorem (see Theorem 5.10 from [15]), there is $f \in L^{1}(\Delta, E)$ such that $f(\xi, \eta) \in \widehat{F}(\xi, \eta, u(\xi, \eta))$ a.e. in $\Delta$. Consequently, $T$ has nonempty values.

Moreover, since from Proposition 3.1 given by Papageorgiou in [11],

$$
\begin{gathered}
\varphi_{\widehat{F}(\cdot, \cdot, u(\cdot, \cdot))}^{1}=\left\{g \in L^{1}(\Delta, E): g(\xi, \eta) \in \widehat{F}(\xi, \eta, u(\xi, \eta))\right\} \\
\in P_{w k c}\left(L^{1}(\Delta, E)\right),
\end{gathered}
$$

$T$ has values in $P_{f c}(C(\Delta, E))$.

Additionally, from (3.15), (3.16), and the first part of assumption (iv), 


$$
\begin{gathered}
v(x, 0)+v(0, y)=\alpha(x, 0)+\alpha(0, y)-\sum_{j=1}^{r} h_{j}(x) u\left(x, b_{j}\right)-\sum_{i=1}^{p} k_{i}(y) u\left(a_{i}, y\right) \\
=u(x, 0)+u(0, y) \in Z, \quad(x, y) \in \Delta
\end{gathered}
$$

if $v \in T(u)$, where $u \in \mathcal{U}$.

Let $B \subset \cup$ be a nonempty set. We have, by (2.1), (3.16), (3.15), (3.18) and (3.17), that

$$
\begin{aligned}
\beta(T(B)(x, y)) \leq \beta\left(\int_{0}^{x} \int_{0}^{y} f(\xi, \eta) d \xi d \eta: f \in \mathcal{\varphi}_{\widehat{F}}^{1}(\cdot, \cdot, u(\cdot, \cdot)), u \in B\right) \\
\leq \beta\left(\int_{0}^{x} \int_{0}^{y} \widehat{F}(\xi, \eta, \overline{B(\xi, \eta)}) d \xi d \eta\right), \quad(x, y) \in \Delta,
\end{aligned}
$$

where

$$
\overline{B(\xi, \eta)}:=\overline{\{u(\xi, \eta): u \in B\}}, \quad(\xi, \eta) \in \Delta,
$$

and

$$
\begin{gathered}
\int_{0}^{x} \int_{0}^{y} \widehat{F}(\xi, \eta, \overline{B(\xi, \eta)}) d \xi d \eta:=\left\{\int_{0}^{x} \int_{0}^{y} h(\xi, \eta) d \xi d \eta: h \in L^{1}(\Delta, E),\right. \\
h(\xi, \eta) \in \widehat{F}(\xi, \eta, \overline{B(\xi, \eta)}) \text { a.e. in } \Delta\},(x, y) \in \Delta .
\end{gathered}
$$

Observe that, for every $x^{*} \in E^{*}, \quad(\xi, \eta, z) \rightarrow \sigma\left(x^{*}, \widehat{F}(\xi, \eta, z)\right)$ is measurable and $(\xi, \eta) \rightarrow \overline{B(\xi, \eta)}$ is graph measurable. Therefore, from the Kandilakis-Papageorgiou theorem (see [9], Theorem 6.1), $(\xi, \eta) \rightarrow \sup \left[\sigma\left(x^{*}, \widehat{F}(\xi, \eta, u)\right): u \in \overline{B(\xi, \eta)}\right]$ is Lebesgue measurable on $\Delta$. Hence, by the third part of assumption $(i),(\xi, \eta) \rightarrow \overline{\text { conv }}$ $\widehat{F}(\xi, \eta, B(\xi, \eta)) \in P_{w k c}(E)$. Consequently, applying an argument given by Papageorgiou in [13] and by Kandilakis-Papageorgiou in [8], we obtain that

$$
\begin{gathered}
\beta\left(\int_{0}^{x} \int_{0}^{y} \widehat{F}(\xi, \eta, \overline{B(\xi, \eta)}) d \xi d \eta\right) \leq \int_{0}^{x} \int_{0}^{y} \mathscr{S}(\xi, \eta) \beta(\overline{B(\xi, \eta)}) d \xi d \eta \\
=\int_{0}^{x} \int_{0}^{y} \mathscr{S}(\xi, \eta) \beta(B(\xi, \eta)) d \xi d \eta, \quad(x, y) \in \Delta .
\end{gathered}
$$

Formulae (3.19)-(3.22) imply that

$$
\beta(T(B)(x, y)) \leq \int_{0}^{x} \int_{0}^{y} \mathscr{S}(\xi, \eta) \beta(B(\xi, \eta)) d \xi d \eta
$$


Let

$$
\leq \int_{0}^{x} \int_{0}^{y}\|\mathscr{G}\|_{\infty} \beta(B(\xi, \eta)) d \xi d \eta, \quad(x, y) \in \Delta
$$

$$
\Psi(B):=\sup _{(\xi, \eta) \in \Delta}\left[e^{-\lambda\|\mathscr{S}\|_{\infty}^{1 / 2}(\xi+\eta)} \beta(B(\xi, \eta))\right]
$$

$$
\text { for } B \subset \mathcal{U} \text { and with } \lambda>0 \text {. }
$$

Since $U \subset C(\Delta, E)$ is bounded and equicontinuous, by properties of $\beta$ and by Arzela-Ascoli theorem, $\Psi$ is a sublinear measure of noncompactness in the sense of Banas-Goebel [1]. Therefore, from (3.23) and (3.24),

$$
\begin{aligned}
& \beta(T(B)(x, y)) \leq \int_{0}^{x} \int_{0}^{y}\|\mathscr{W}\|_{\infty} e^{\lambda\|\mathscr{W}\|_{\infty}^{1 / 2}(\xi+\eta)} e^{-\lambda\|\mathscr{W}\|_{\infty}^{1 / 2}(\xi+\eta)} \beta(B(\xi, \eta)) d \xi d \eta \\
& \leq \int_{0}^{x} \int_{0}^{y}\|\mathscr{S}\|_{\infty} e^{\lambda\|\mathscr{S}\|_{\infty}^{1 / 2}(\xi+\eta)} \Psi(B) d \xi d \eta \leq \frac{\Psi(B)}{\lambda^{2}} e^{\lambda\|\mathscr{S}\|_{\infty}^{1 / 2}(x+y)},(x, y) \in \Delta
\end{aligned}
$$

By (3.25) and (3.24),

$$
\Psi(T(B)) \leq \frac{1}{\lambda^{2}} \Psi(B) .
$$

The above inequality implies that $T$ is a $\Psi$ contraction if $\lambda>1$.

Next, we will show that the graph of $T$

$$
\operatorname{Gr} T:=\{(u, v) \in \mathcal{U} \times \mathcal{U}: \quad v \in T(u)\}
$$

is closed in $C(\Delta, E) \times C(\Delta, E)$.

To this end, let $\left(u_{n}, v_{n}\right) \in \operatorname{Gr} T(n=1,2, \ldots)$ and assume that

$$
\left(u_{n}, v_{n}\right)_{n \rightarrow \infty}(u, v) \text { in } \cup \times \cup \subset C(\Delta, E) \times C(\Delta, E) .
$$

We know that functions $u_{n}$ and $v_{n}(n=1,2, \ldots)$ satisfy the following conditions:

$$
\begin{gathered}
u_{n}(x, 0)+u_{n}(0, y) \in Z, \quad(x, y) \in \Delta \quad(n=1,2, \ldots), \\
v_{n}(x, 0)+v_{n}(0, y) \in Z, \quad(x, y) \in \Delta \quad(n=1,2, \ldots), \\
v_{n}(x, y)=\alpha(x, y)-\sum_{j=1}^{r} h_{j}(x) u_{n}\left(x, b_{j}\right)-\sum_{i=1}^{p} k_{i}(y) u_{n}\left(a_{i}, y\right) \\
+\int_{0}^{x} \int_{0}^{y} g_{n}(\xi, \eta) d \xi d \eta, \quad(x, y) \in \Delta \quad(n=1,2, \ldots),
\end{gathered}
$$


with $g_{n} \in L^{1}(\Delta, E), g_{n}(\xi, \eta) \in \widehat{F}\left(\xi, \eta, u_{n}(\xi, \eta)\right)(n=1,2, \ldots)$ a.e. in $\Delta$.

Since $\widehat{F}(\xi, \eta, \cdot)$ is upper semicontinuous from $E$ into $E_{w}$ with values in $P_{k c}(E)$, then, applying Theorem 7.4 .2 from [10],

$$
(\xi, \eta) \rightarrow \overline{\operatorname{conv}} \bigcup_{n \in \mathbb{N}} \widehat{F}\left(\xi, \eta, u_{n}(\xi, \eta)\right)=: G(\xi, \eta)
$$

is a measurable, $P_{w k c}(E)$-valued multifunction such that

$$
|G(\xi, \eta)|:=\sup \{\|s\|: s \in G(\xi, \eta)\} \leq c_{3}(\xi, \eta) \text { a.e. in } \Delta .
$$

Consequently, from [11], we have that

$$
\varphi_{G}^{1}:=\left\{g \in L^{1}(\Delta, E): g(\xi, \eta) \in G(\xi, \eta) \text { a.e. in } \Delta\right\}
$$

is weakly compact in $L^{1}(\Delta, E)$. Thus, passing to a subsequence if necessary, we may assume that

$$
g_{n} \underset{n \rightarrow \infty}{\stackrel{w}{\rightrightarrows}} g \text { in } L^{1}(\Delta, E)
$$

By the fact that $z \rightarrow \widehat{F}(x, y, z)$ is upper semicontinuous from $E$ into $E_{w}$, by the convergence of $u_{n}$ to $u$ in $C(\Delta, E)$ and by an argument given by Papageorgiou in [12, $13]$,

$$
g(\xi, \eta) \in \widehat{F}(\xi, \eta, u(\xi, \eta)) \text { a.e. in } \Delta .
$$

Therefore, from (3.27) and (3.29),

$$
\begin{aligned}
v(x, y) & =\alpha(x, y)-\sum_{j=1}^{r} h_{j}(x) u\left(x, b_{j}\right)-\sum_{i=1}^{p} k_{i}(y) u\left(a_{i}, y\right) \\
& +\int_{0}^{x} \int_{0}^{y} g(\xi, \eta) d \xi d \eta, \quad(x, y) \in \Delta,
\end{aligned}
$$

with $g \in L^{1}(\Delta, E)$ and $g(\xi, \eta) \in \widehat{F}(\xi, \eta, u(\xi, \eta))$ a.e. in $\Delta$.

Moreover, by (3.27) and (3.28),

and

$$
u(x, 0)+u(0, y) \in Z \text { for }(x, y) \in \Delta
$$

$$
v(x, 0)+v(0, y) \in Z \text { for }(x, y) \in \Delta \text {. }
$$

So $(u, v) \in \operatorname{Gr} T$ and, consequently, $T$ has a closed graph in $U \times \mathcal{U} \subset C(\Delta, E) \times$ $C(\Delta, E)$.

Inequality (3.26) and the fact that $\operatorname{Gr} T$ is closed imply, by the Tarafdar-Vyborny theorem (see [14], Theorem 4.1), that $u \in T(u)$.

As in the beginning of the proof of Theorem 3.1, applying the definition of $\widehat{F}$ and Gronwall's inequality, we obtain that

$$
\|u\|_{C(\Delta, E)} \leq M
$$

where $M$ is given by formula (3.10). 
Therefore,

$$
\widehat{F}(x, y, u(x, y))=F(x, y, u(x, y)), \quad(x, y) \in \Delta .
$$

Consequently, $u \in K(\Delta, E)$ is a solution of problem (2.2).

The proof of Theorem 3.1 is complete.

\section{Theorem About the Existence of a Solution of the Nonlocal Multi- valued Darboux Problem with the Nonconvex Valued Orientor Field}

Theorem 4.1: Suppose that $F: \Delta \times E \rightarrow P_{f}(E)$ is a multifunction such that:

(a) $\quad(x, y, z) \rightarrow F(x, y, z)$ is measurable, $z \rightarrow F(x, y, z)$ is lower semicontinuous and for all $(x, y) \in \Delta, F(x, y, \cdot)$ maps the bounded sets into relatively weakly compact sets,

(b) assumptions (ii)-(iv) of Theorem 3.1 are satisfied.

Then in a class of functions $w \in K(\Delta, E)$ problem $(2.2)$ possesses a solution.

Proof: As in the proof of Theorem 3.1, if $u \in C(\Delta, E)$ is a solution of problem (2.2) then

$$
\|u(x, y)\| \leq M, \quad(x, y) \in \Delta
$$

where $M$ is given by $(3.10)$.

Define $\widehat{F}: \Delta \times E \rightarrow P_{f}(E)$ by formula (3.11). Consequently,

$$
\widehat{F}(x, y, z)=F\left(x, y, p_{M}(z)\right) \text { in } \Delta \times E,
$$

where $p_{M}$ in the $M$-radial retraction in $E$, and Theorem 7.3.11 from [10] implies that $z \rightarrow \widehat{F}(x, y, z)$ is lower semicontinuous.

Introduce a set $\mathcal{U}$ by the formula

$$
\begin{gathered}
\mathcal{U}:=\{u \in C(\Delta, E): u(x, 0)+u(0, y) \in Z, \quad(x, y) \in \Delta, \\
u(x, y)=\alpha(x, y)-\sum_{j=1}^{r} h_{j}(x) u\left(x, b_{j}\right)-\sum_{i=1}^{p} k_{i}(y) u\left(a_{i}, y\right) \\
+\int_{0}^{x} \int_{0}^{y} g(\xi, \eta) d \xi d \eta, \quad(x, y) \in \Delta, \\
\left.\|g(\xi, \eta)\| \leq c_{3}(x, y) \text { a.e. in } \Delta\right\} .
\end{gathered}
$$

The above set is a nonempty, bounded, closed an equicontinuous subset of $C(\Delta, E)$.

Let $\Gamma: \mathcal{U} \rightarrow P_{f}\left(L^{1}(\Delta, E)\right)$ be the multifunction defined by

$$
\Gamma(u):=\varphi_{\widehat{F}(\cdot, \cdot, u(\cdot, \cdot))}^{1}=\left\{g \in L^{1}(\Delta, E): g(\xi, \eta) \in \widehat{F}(\xi, \eta, u(\xi, \eta)),(\xi, \eta) \in \Delta\right\}
$$




$$
u \in \mathcal{U} \text {. }
$$

The Papageorgiou theorem (see [12], Theorem 4.1) implies that $\Gamma$ is lower semicontinuous. Therefore, from the Bressan-Colombo theorem (see [2], Theorem 3), there is a continuous mapping $\gamma: \mathcal{U} \rightarrow L^{1}(\Delta, E)$ such that $\gamma(u) \in \Gamma(u)$ for all $u \in \mathcal{U}$.

Let

$$
\begin{gathered}
\mu(u)(x, y):=\alpha(x, y)-\sum_{j=1}^{r} h_{j}(x) u\left(x, b_{j}\right)-\sum_{i=1}^{p} k_{i}(y) u\left(a_{i}, y\right) \\
+\int_{0}^{x} \int_{0}^{y} \gamma(u)(\xi, \eta) d \xi d \eta, \quad u \in \mathcal{U},(x, y) \in \Delta .
\end{gathered}
$$

Then $\mu: \mathcal{U} \rightarrow \mathcal{U}$ and, by the continuity of $\gamma, \mu$ is continuous.

Let $B$ be a nonempty, bounded and closed subset of $\mathcal{U}$. Moreover, let $\left\{u_{n}\right\}_{n \in \mathbb{N}}$ be a sequence such that $\left\{u_{n}\right\}_{n \in \mathbb{N}} \subset B$ and ${\overline{\left\{u_{n}\right\}_{n \in \mathbb{N}}}}^{C(\Delta, E)}=B$. We have, from (2.1), (4.2), (4.1), (3.18) and (3.2), that

$$
\begin{gathered}
\beta(\mu(B)(x, y)) \leq \beta\left(\int_{0}^{x} \int_{0}^{y} \gamma(B)(\xi, \eta) d \xi d \eta\right) \\
\leq \beta\left(\int_{0}^{x} \int_{0}^{y} \gamma\left(\left\{u_{n}\right\}_{n \in \mathbb{N}}\right)(\xi, \eta) d \xi d \eta\right) \leq \int_{0}^{x} \int_{0}^{y}\|\mathscr{S}\|_{\infty} \beta\left(\left\{u_{n}(\xi, \eta)\right\}_{n \in \mathbb{N}}\right) d \xi d \eta \\
=\int_{0}^{x} \int_{0}^{y}\|\mathscr{S}\|_{\infty} \beta(B(\xi, \eta)) d \xi d \eta, \quad(x, y) \in \Delta .
\end{gathered}
$$

Define the sublinear measure of noncompactness $\Psi$ by formula (3.24). Arguing as in the proof of Theorem 3.1, we obtain

$$
\Psi(\mu(B)) \leq \frac{1}{\lambda^{2}} \Psi(B) .
$$

The above inequality implies that $\mu$ is a $\Psi$ contraction if $\lambda>1$.

Inequality (4.4) and the Tarafdar-Vyborny theorem (see [14], Theorem 4.1) imply that $u=\mu(u)$ for some $u \in \mathcal{U}$.

As in the beginning of the proof of Theorem 3.1, we obtain inequality (3.30). Therefore, (3.31) holds and $u \in K(\Delta, E)$ is a solution of problem (2.2).

The proof of Theorem 4.1 is complete.

\section{References}

[1] Banas, J. and Goebel, K., Measures of Noncompactness in Banach Spaces, Lect. Notes in Pure and Appl. Math. 60, Marcel Dekker, New York 1980. 
[2] Bressan, A. and Colombo, G., Extensions and selections of maps with decomposable values, Studia Math. 90 (1988), 69-86.

[3] Byszewski, L., Existence and uniqueness of solutions of nonlocal problems for hyperbolic equation $u_{x t}=F\left(x, t, u, u_{x}\right)$, J. of Appl. Math. and Stoch. Anal. 3:3 (1990), 163-168.

[4] Byszewski, L., Theorem about existence and uniqueness of continuous solution of nonlocal problem for nonlinear hyperbolic equation, Appl. Anal. 40 (1991), 173-180.

[5] Byszewski, L. and Lakshmikantham, V., Monotone iterative technique for nonlocal hyperbolic differential problem, J. of Math. and Phys. Sci. 26:4 (1992),345-359.

[6] Byszewski, L., Differential and Functional-Differential Problems with Nonlocal Conditions, Cracow University of Technology Monograph 184, Cracow 1995.

[7] Dawidowski, M. and Kubiaczyk, I., An existence theorem for the generalized hyperbolic equation $z_{x y}^{\prime \prime} \in F(x, y, z)$ in Banach space, Annales Soc. Math. Polonae, Comment. Math. 30 (1990), 41-49.

[8] Kandilakis, D. and Papageorgiou, N.S., On the properties of the Aumann integral with applications to differential inclusions and optimal control systems, Czech. Math. J. 39 (1989), 1-15.

[9] Kandilakis, D. and Papageorgiou, N.S., Properties of measurable multifunctions with stochastic domain and their applications, Math. Japonica 35 (1990), 629643.

[10] Klein, E. and Thompson, A., Theory of Correspondence, Wiley, New York 1984.

[11] Papageorgiou, N.S., On the theory of Banach space valued multifunctions, Part 1: Integration and conditional expectation, J. Multiv. Anal. 17 (1985), 185-206.

[12] Papageorgiou, N.S., Convergence theorems for Banach space valued integrable multifunctions, Intern. J. Math. and Math. Sci. 10 (1987), 433-442.

[13] Papageorgiou, N.S., Existence of solutions for hyperbolic differential inclusions in Banach spaces, Archivum Math. (Brno) 28 (1992), 205-213.

[14] Tarafdar, E. and Vyborny, R., Fixed point theorems for condensing multivalued mappings on a locally convex topological space, Bull. Austr. Math. Soc. 12 (1975), 161-170.

[15] Wagner, D., Survey of measurable selection theorems, SIAM J. Control and Optim. 15 (1977), 859-903. 


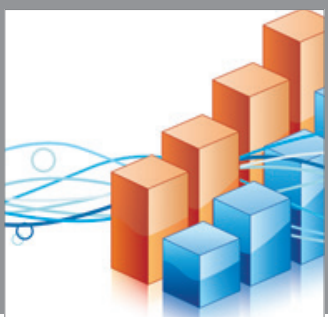

Advances in

Operations Research

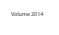

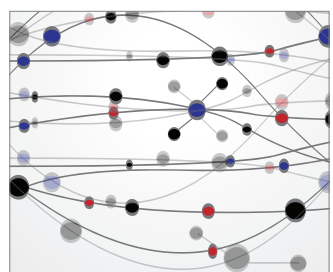

\section{The Scientific} World Journal
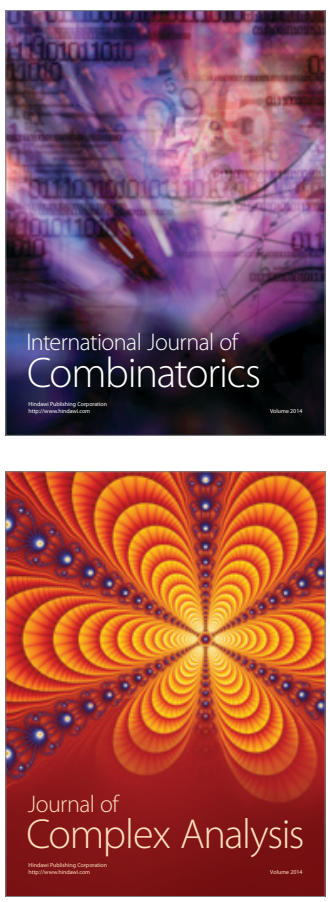

International Journal of

Mathematics and

Mathematical

Sciences
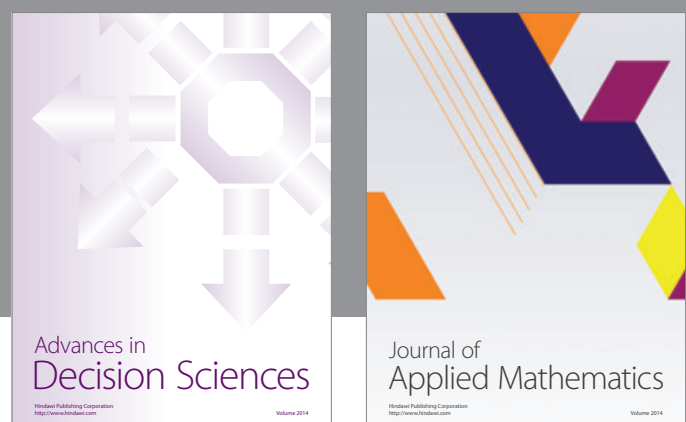

Journal of

Applied Mathematics
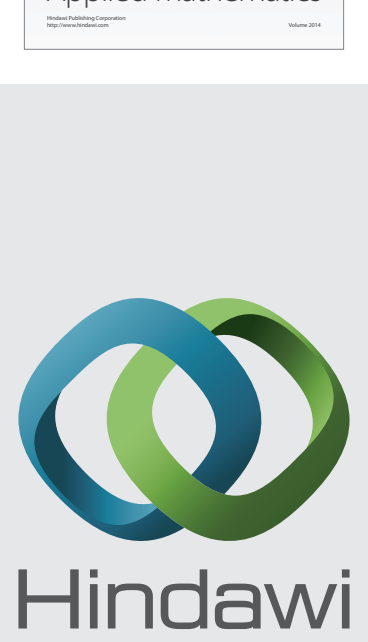

Submit your manuscripts at http://www.hindawi.com
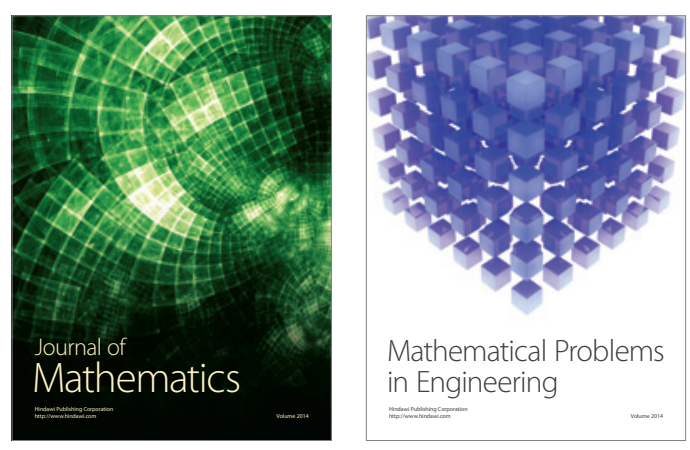

Mathematical Problems in Engineering
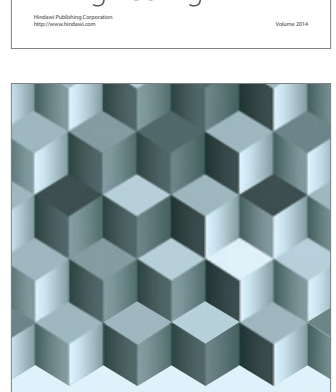

Journal of

Function Spaces
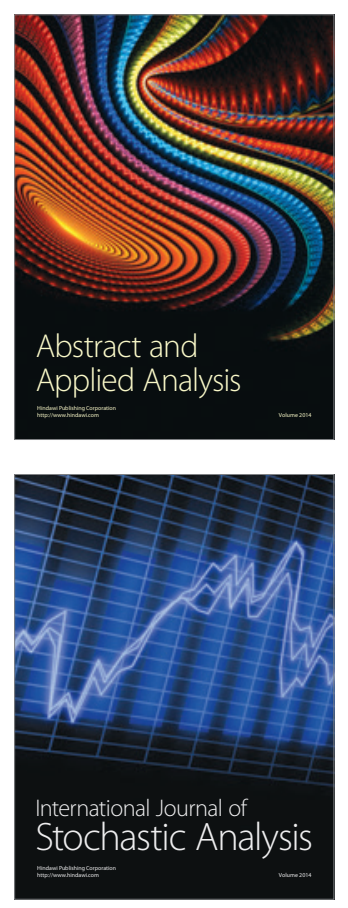

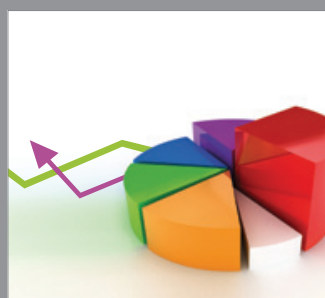

ournal of

Probability and Statistics

Promensencen
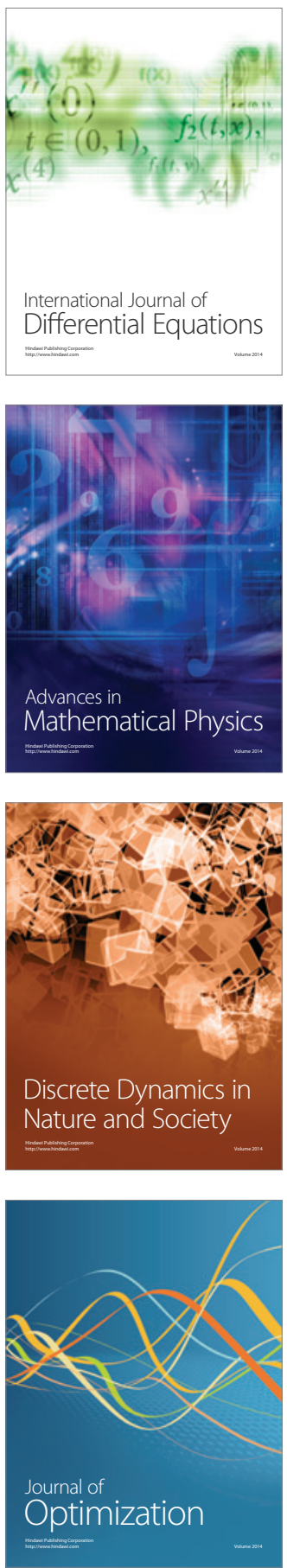